\title{
JOURNAL OF HYDRODYNAMICS
}

Accepted version published online 30 June 2016

DOI: 10.1016/S1001-6058(16)60649-2

\section{NUMERICAL SOLUTION OF THERMO-SOLUTAL MIXED CONVECTIVE SLIP FLOW PAST A RADIATIVE PLATE WITH CONVECTIVE BOUNDARY CONDITION}

\author{
M. J. UDDIN ${ }^{*}$, O. ANWAR BÉG ${ }^{2}$, M. N. UDDIN ${ }^{3}$, A. I. Md. ISMAIL ${ }^{4}$ \\ 1. Department of Mathematics, American International University-Bangladesh, Dhaka, Bangladesh, \\ 2. Fluid Mechanics, Spray Research Group, Petroleum and Gas Engineering Division, Room G77, Newton Building, \\ School of Computing, Science and Engineering (CSE), University of Salford, M54WT, UK \\ 3. Department of Mathematical Sciences, Ball State University, 2000 W University Avenue, Muncie, IN 47306, USA \\ 4. School of Mathematical Sciences, Universiti Sains Malaysia, Penang 11800, Malaysia
}

\begin{abstract}
A mathematical model for mixed convective slip flow with heat and mass transfer in the presence of thermal radiation is presented. A convective boundary condition is included and slip is simulated via the hydrodynamic slip parameter. Heat generation or absorption effects are also incorporated. The Rosseland diffusion flux model is employed. The governing partial differential conservation equations are reduced to a system of coupled, ordinary differential equations via Lie group theory methods. The resulting coupled equations are solved using shooting method. The influences of the emerging parameters on dimensionless velocity, temperature and concentration distributions are investigated. Increasing radiative-conductive parameter accelerates the boundary layer flow and increase temperatures whereas it depresses concentration. An elevation in convection-conduction parameter also accelerates the flow and temperatures whereas it reduces concentrations. Velocity near the wall is considerably boosted with increasing momentum slip parameter although both temperature and concentration boundary layer thicknesses are decreased. The presence of a heat source is found to increase momentum and thermal boundary layer thicknesses but reduces concentration boundary layer thickness. Excellent correlation of the numerical solutions with previous non-slip studies is demonstrated. The current study has applications in bio-reactor diffusion flows and hightemperature chemical materials processing systems.
\end{abstract}

Keywords: Slip flow, Thermal radiation, Convective heat and mass transfer, Lie Group theory, heat source/sink, materials processing.

*Corresponding author-email: jashim_74@yahoo.com 


\section{INTRODUCTION}

Slip flows occurs in a diverse range of technological applications including sheet processing ${ }^{[1]}$, nuclear power systems ${ }^{[2]}$, and foam production ${ }^{[3]}$, fluidic cells in medicine ${ }^{[4]}$. Slip flows require a modification of the classical "no-slip" velocity boundary condition at a wall with a "slip boundary condition" and have stimulated considerable interest in recent years. Ulmanella and Ho ${ }^{[5]}$ found via experiments, the velocity for various micro-sized channels. The velocity is a function of shear rate, type of liquid and surface morphology. The possibility of temperature jump along with velocity slip was described by Bocquet and Barrat ${ }^{[6]}$. The velocity slip and temperature jump was modelled by Bocquet and Barrat ${ }^{[6]}$ via the introduction of velocity slip length and temperature slip length terms, respectively. Many analytical and numerical methods have been employed to simulate a wide spectrum of slip flows. Nandeppanavar et al. ${ }^{[7]}$ studied analytically and numerically the effects of first and second order slip parameters on thermal convection boundary layer flow from a stretching sheet. Turkyilmazoglu ${ }^{[8]}$ investigated analytically the influence of hydrodynamic and thermal slip conditions on double-diffusive magnetic convection of a nanofluid. Tripathi et al. ${ }^{[9]}$ used a homotopy semi-computational method to study peristaltic slip flow of a non-Newtonian fluid, showing that pressure difference is strongly reduced with increasing wall slip effect. Wang ${ }^{[10]}$ reported on the interaction of surface (momentum) slip on rotating Poiseuille and Couette flows, showing that for the former case slip enhances longitudinal flow rate at low rotation, but decreases it at high rotation, whereas in the latter case, longitudinal drag is reduced with greater slip. Mahmoud ${ }^{[11]}$ employed a Chebyshev spectral numerical code to study velocity slip and variable thermophysical property effects on stagnation-point flow in a porous regime. Taamneh and Omari ${ }^{[12]}$ examined the slipflow and heat transfer in non-Newtonian inelastic fluids in a porous medium micro-channel numerically. Anand [13] presented slip effects on heat transfer for power law fluid in a microchannel which is subjected to constant heat flux boundary condition. Jamalabadi et al. ${ }^{[14]}$ studied combined convection flow in a vertical channel with slip boundary conditions using an entropy generation minimization method. Abbas et al. ${ }^{[15]}$ studied stagnation-point flow of a hydromagnetic viscous fluid past stretching/shrinking sheet taking into account slip boundary condition. Water slip flow in superhydrophobic microtubes within laminar flow region was examined by Yu et al. ${ }^{[16]}$. Nandy ${ }^{[17]}$ studied unsteady flow of Maxwell fluid toward a permeable shrinking surface with Navier slip. Haq et al. ${ }^{[18]}$ studied thermal slip effects on MHD stagnation 
point flow of nanofluid past a stretchable sheet. Effects of hydrodynamic slip on the steady flow of an incompressible electrically conducting fluid past a channel with porous plates are reported by Ibáñez ${ }^{[19]}$.

Double-diffusive (thermo-solutal) convection flows are also of interest in many branches of engineering sciences including energy storage ${ }^{[20]}$, nuclear reactor leakage hazards ${ }^{[21]}$, nano-

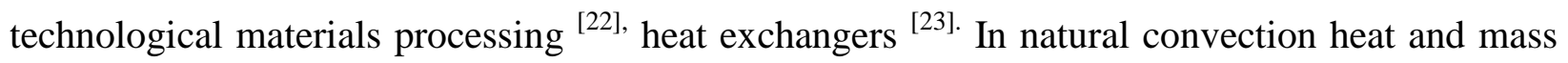
transfer, thermal and species buoyancy effects play a significant role. Slip flows with combined heat and mass transfer have also received some attention, primarily in chemical engineering applications. Das ${ }^{[24]}$ investigated partial slip and thermal radiation effects on magnetized nonNewtonian heat and mass transfer from an inclined surface.

Thermal radiation heat transfer also arises in many chemical engineering processes. At a high temperature, thermal radiation may change the distribution of temperature in the boundary layer and this affects the heat transfer at the wall. A variety of radiative heat transfer models have been utilized for transport modelling. Owing to the complexity of solving the integro-differential equation of radiative transfer these models are frequently algebraic flux approximations [ ${ }^{25]}$. Radiative flux was observed to significantly modify the critical Rayleigh and wave numbers and affect convection strongly. Mahmoud ${ }^{[26]}$ employed the Rosseland diffusion flux model to study mixed convection-radiation flow from a horizontal permeable surface aligned parallel to a uniform free stream. Gupta et al. ${ }^{[27]}$ used a variational finite element method and Rosseland flux model to simulate non-Newtonian radiative-convection flow from a shrinking polymeric sheet showing that the flow is accelerated substantially with increasing thermal radiation effect which also supplements energy transfer to the flow and enhances thermal boundary layer thickness. The Rosseland diffusion model was also implemented by Khan et al. ${ }^{[28]}$ for radiative nanofluid slip flow. Hussain et al. ${ }^{[29]}$ presented homotopy solution of boundary layer flow of a micropolar fluid towards a porous stretchable sheet. Mohan Krishna et al. ${ }^{[30]}$ studied radiation effects on MHD flow over a moving plate. Srinivasacharya and Reddy ${ }^{[31]}$ investigated radiation effects on mixed convection heat and mass transfer over a flat plate in porous medium filled with power-law fluid. An experimental work on mixed convection and radiation heat transfer in a horizontal duct with variable wall temperature was presented by Ganesan et al. ${ }^{[32]}$. Ashraf et al. ${ }^{[33]}$ studied 
convective heat and mass transfer in MHD mixed convection flow of Jeffrey nanofluid over a radially stretching surface with thermal radiation. Mixed convection-radiation on stagnationpoint flow of nanofluids past a stretching/shrinking sheet in a porous medium was investigated by $\mathrm{Pal}$ and Mandal ${ }^{[34]}$.

In the present study a mathematical model is developed for buoyancy-driven mixed thermal convection-radiation slip flow from a vertical plate with species diffusion. A convective surface boundary condition is also prescribed and heat source/sink effects included. The Rosseland diffusion flux model is used for radiative heat transfer. Via Lie group theory methods the conservation equations are rendered into similarity ordinary differential equations and solutions developed with numerical shooting method. Selected solutions are verified with comparison to earlier non-slip flows.

\section{MATHEMATICAL FLOW MODEL}

Newtonian viscous incompressible double-diffusive mixed convection is studied. The geometry and the rectangular coordinates, $\bar{x}$ and $\bar{y}$, and the corresponding velocity components, $\bar{u}$ and $\bar{v}$ and flow configuration are illustrated in Fig. 1 (in which $i$ represents momentum while $i i$ represents thermal and concentration boundary layers; in general thermal and concentration boundary layer thickness are not the same). It is assumed that the uniform temperature of the ambient fluid is $T_{\infty}$, the unknown temperature of the plate is $T_{w}$ and the left surface of the plate is heated from a hot fluid of temperature $T_{f}\left(>T_{\infty}\right)$ or is cooled from a cooled fluid $\left(T_{f}<T_{\infty}\right)$ by the process of convection. This then yields a heat transfer variable coefficient $h_{f}(\bar{x} / L)$. It is assumed that the thermal radiation is present in the form of a uni-directional flux, applied transverse to the wall surface and obeys the Rosseland diffusion approximation. This formulation allows the transformation of the governing integro-differential equation for radiative energy balance into a Fourier-type diffusion equation analogous to that describing heat conduction or electrostatic potential (Coulomb's law). The model is generally applicable for optically-thick media in which radiation is only known to propagate a limited distance prior to experiencing scattering or absorption. Implicit in this approach is the assumption that refractive index of the medium is constant, intensity within the fluid is nearly isotropic and uniform and wavelength 
regions exist where the optical thickness is greater than five. It is further assumed that the concentration (low) of the ambient fluid is the uniform concentration $C_{\infty}$, the unknown concentration of the plate is $C_{w}\left(>C_{\infty}\right)$. Fluid properties are assumed to be invariant except density, which is assumed to vary only in those changes that drive the flow (i.e., the Boussinesq approximation). A heat source (or sink) is also present. The governing boundary layer equations based on scale analysis in dimensional form relevant to our problem may be presented as follows $\left(\right.$ Bejan $^{[35]}$ ):

$\frac{\partial \bar{u}}{\partial \bar{x}}+\frac{\partial \bar{v}}{\partial \bar{y}}=0$

$\rho\left(\bar{u} \frac{\partial \bar{u}}{\partial \bar{x}}+\bar{v} \frac{\partial \bar{u}}{\partial \bar{y}}\right)=\rho \bar{u}_{e} \frac{d \bar{u}_{e}}{d \bar{x}}+\mu \frac{\partial^{2} \bar{u}}{\partial \bar{y}^{2}}+\rho g \beta_{T}\left(T-T_{\infty}\right)+\rho g \beta_{C}\left(C-C_{\infty}\right)$,

$\bar{u} \frac{\partial T}{\partial \bar{x}}+\bar{v} \frac{\partial T}{\partial \bar{y}}=\left(\alpha+\frac{16 \sigma_{1} T_{\infty}^{3}}{3 \rho c_{p} k_{1}}\right) \frac{\partial^{2} T}{\partial \bar{y}^{2}}+\frac{Q(\bar{x})}{\rho c_{p}}\left(T-T_{\infty}\right)$

$\bar{u} \frac{\partial C}{\partial \bar{x}}+\bar{v} \frac{\partial C}{\partial \bar{y}}=D \frac{\partial^{2} C}{\partial \bar{y}^{2}}$

Following Karniadakis et al. ${ }^{[36]}$ to get physically realistic results, we adopt velocity slip boundary condition at the wall and hence the boundary conditions at the wall are:

$$
\bar{v}=0, \bar{u}=N_{1}\left(\frac{\bar{x}}{L}\right) v \frac{\partial \bar{u}}{\partial \bar{y}}, \quad-k \frac{\partial T}{\partial \bar{y}}=h_{f}\left(\frac{\bar{x}}{L}\right)\left[T_{f}-T_{w}\right], C=C_{w}, \text { at } \quad \bar{y}=0,
$$

Based on problem description, the far filed boundary conditions are

$$
\bar{u} \rightarrow \bar{u}_{e}=\lambda\left(\frac{\bar{x}}{L}\right)^{1 / 2} \bar{u}_{r}, \quad T \rightarrow T_{\infty}, \quad C \rightarrow C_{\infty} \quad \text { as } \quad \bar{y} \rightarrow \infty
$$

where $T$ is the temperature $C$ is the concentration, $k$ is the thermal conductivity, $D$ is the mass diffusivity of species of the fluid, $\beta_{T}$ is the volumetric thermal coefficient, $\beta_{C}$ is the volumetric concentration coefficient, $g$ is the acceleration due to gravity, $\sigma_{1}$ is the Stefan- 
Boltzmann constant, $k_{1}$ is the Rosseland mean absorption coefficient, $\alpha=\frac{k}{\rho c_{p}}$ is the thermal diffusivity of the fluid, $\rho$ is the density of the fluid, $\mu$ is the viscosity of the fluid, $h_{f}(\bar{x} / L)$ is the heat transfer coefficient, $N_{1}$ is the velocity slip factor, $Q(\bar{x})=Q_{0} /(\bar{x} / L)^{1 / 2}$ is the volumetric heat generation or absorption, $\lambda=0$ stand for stationary free stream (purely free convection) whilst $\lambda=1$ stand for moving free stream (mixed convection).

\subsection{TRANSFORMATION OF MODEL AND LIE GROUP ANALYSIS}

We introduce the following boundary layer variables to transform Eqns. (1)-(5) to dimensionless form.

$x=\frac{\bar{x}}{L}, y=\frac{\bar{y} \mathrm{Re}^{1 / 2}}{L}, u=\frac{\bar{u}}{U_{r}}, v=\frac{\bar{v} L}{v \operatorname{Re}^{1 / 2}}, u_{e}=\frac{\bar{u}_{e}}{U_{r}}, \theta=\frac{T-T_{\infty}}{T_{f}-T_{\infty}}, \phi=\frac{C-C_{\infty}}{C_{w}-C_{\infty}}$,

where $\operatorname{Re}=\frac{U_{r} L}{v}$ is the Reynolds number based on the characteristic length $L$, $U_{r}=\sqrt{g \beta_{T}\left(T_{f}-T_{\infty}\right) L}$ is the reference velocity. We introduce the dimensional stream function $\psi$ defined as $u=\frac{\partial \psi}{\partial y}, v=-\frac{\partial \psi}{\partial x}$ into Eqns. (2)-(4) to reduce the number of equations and dependent variables. This leads to the following three dimensionless partial differential equations for momentum, heat and species conservation.

$$
\begin{aligned}
& \frac{\partial \psi}{\partial y} \frac{\partial^{2} \psi}{\partial x \partial y}-\frac{\partial \psi}{\partial x} \frac{\partial^{2} \psi}{\partial y^{2}}-\frac{\partial^{3} \psi}{\partial y^{3}}-[\theta+N \phi]-\frac{\lambda^{2}}{2}=0 \\
& \operatorname{Pr}\left(\frac{\partial \psi}{\partial y} \frac{\partial \theta}{\partial x}-\frac{\partial \psi}{\partial x} \frac{\partial \theta}{\partial y}\right)-(1+N r) \frac{\partial^{2} \theta}{\partial y^{2}}-\frac{\operatorname{Pr}}{x^{1 / 2}} G \theta=0 \\
& S c\left(\frac{\partial \psi}{\partial y} \frac{\partial \phi}{\partial x}-\frac{\partial \psi}{\partial x} \frac{\partial \phi}{\partial y}\right)-\frac{\partial^{2} \phi}{\partial y^{2}}=0
\end{aligned}
$$

Here $\operatorname{Pr}=v / \alpha$ is the Prandtl number, $S c=v / D$ is the Schmidt number, $N=\beta_{C}\left(C_{w}-C_{\infty}\right) / \beta_{T}\left(T_{f}-T_{\infty}\right)$ is the buoyancy ratio parameter, $(N>0$ corresponds to 
buoyancy-aiding flow and $N<0$ to buoyancy-opposing flow), $N r=16 \sigma_{1} T_{\infty}^{3} / 3 k_{1} k$ is the radiation-conduction parameter and $G=\frac{Q_{0} L}{\rho C_{p} U_{r}}$ is the internal heat generation (source)/absorption (sink) parameter.

The transformed boundary conditions in Eqn. (5) become:

$$
\begin{aligned}
& \frac{\partial \psi}{\partial y}=\frac{\operatorname{Re}^{1 / 2} N_{1}(x) v}{L} \frac{\partial^{2} \psi}{\partial y^{2}}, \frac{\partial \psi}{\partial x}=0, \frac{\partial \theta}{\partial y}=-\frac{L h_{f}(x)}{k \operatorname{Re}^{1 / 2}}(1-\theta), \phi=1 \text { at } y=0, \\
& \frac{\partial \psi}{\partial y} \rightarrow \lambda \sqrt{x}, \theta \rightarrow 0, \phi \rightarrow 0 \text { as } \quad y \rightarrow \infty .
\end{aligned}
$$

The transformed boundary layer equations and boundary conditions i.e. Eqns. (7)-(10) constitute a strongly coupled, highly nonlinear boundary value problem. Analytical solutions are extremely difficult. To obtain direct numerical solutions of these equations are also complicated and computationally expensive. Similarity solutions have proven to be an efficient tool to solve various transport problems. In view of this, we implement a linear group of transformations which combines the two independent variables $(x, y)$ into a single independent variable $\eta$ (similarity variable) and reduce Eqns. (7)-(10) into a system of coupled ordinary differential equations with the corresponding boundary conditions. Group theory methods have proved an efficient and very useful in recent years and have been successfully used to a wide range of complex flows in chemical engineering. These include nanofluid transport in porous media ${ }^{\text {[37] }}$ bioconvective flow ${ }^{[38]}$, chemically-reacting boundary layer flows ${ }^{[39]}$, rotating disk problem ${ }^{\text {[40], }}$ MHD flow ${ }^{[41]}$. In accordance with the group theory methodology, we scale all independent and dependent variables as follows:

$$
x^{*}=x A^{\alpha_{1}}, y^{*}=y A^{\alpha_{2}}, \psi^{*}=\psi A^{\alpha_{3}}, \theta^{*}=\theta A^{\alpha_{4}}, \phi^{*}=\phi A^{\alpha_{5}}, h_{f}^{*}=h_{f} A^{\alpha_{6}}, N_{1}^{*}=N_{1} A^{\alpha_{7}}
$$

where $A, \alpha_{i}(i=1,2, \ldots, 6,7)$ are constants. We seek the values of $\alpha_{i}$ such that the forms of the Eqns. (7)-(10) are invariant under the transformations. Eqns. (7)-(10) will be invariant if $\alpha_{i}$ are satisfies the flowing relations 
$\alpha_{1}=4 \alpha_{2}, \alpha_{3}=3 \alpha_{2}, \alpha_{4}=\alpha_{5}=0, \alpha_{6}=-\alpha_{2}, \alpha_{7}=\alpha_{2}$.

Now, we seek "absolute invariants" (functions having the same form before and after the transformation under this group of transformations ${ }^{[35-39}$. An inspection of Eqns. (11) and (12) that revealed that

$$
\frac{y}{x^{1 / 4}}=\frac{y^{*}}{x^{* 1 / 4}}
$$

This combination of variables is therefore invariant under this group of transformations and hence, is an absolute invariant. We denote this functional form as:

$$
\eta=\frac{y}{\sqrt[4]{x}}
$$

Using the same argument, the other absolute invariants are:

$$
\psi=x^{3 / 4} f(\eta), \theta=\theta(\eta), \phi=\phi(\eta), h_{f}=x^{-1 / 4}\left(h_{f}\right)_{0}, N_{1}=x^{1 / 4}\left(N_{1}\right)_{0},
$$

where $\eta$ is the similarity independent variable, $f(\eta), \theta(\eta)$ and $\phi(\eta)$ are respectively the dimensionless velocity, temperature and concentration functions, $\left(h_{f}\right)_{0}$ is the constant heat transfer coefficient and $\left(N_{1}\right)_{0}$ is the constant hydrodynamic slip factor.

\subsection{Governing similarity equations}

Substituting Eqns. (14) and (15) into Eqns. (7) - (9), generates the following ordinary differential equations:

$$
\begin{aligned}
& f^{\prime \prime \prime}+\frac{1}{4}\left(3 f f^{\prime \prime}-2 f^{\prime 2}\right)+\theta+N \phi+\frac{\lambda^{2}}{2}=0, \\
& (1+N r) \theta^{\prime \prime}+\frac{3 \operatorname{Pr}}{4} f \theta^{\prime}+\operatorname{Pr} G \theta=0, \\
& \phi^{\prime \prime}+\frac{3 S c}{4} f \phi^{\prime}=0
\end{aligned}
$$


subject to the boundary conditions:

$f(0)=0, f^{\prime}(0)=a f^{\prime \prime}(0), \theta^{\prime}(0)=-N c[1-\theta(0)], \phi(0)=1, f^{\prime}(\infty)=\lambda, \theta(\infty)=\phi(\infty)=0$,

where primes denote differentiation with respect to $\eta$. Here $N c=\left(h_{f}\right)_{0} L / k \operatorname{Re}^{1 / 2}$ is the convection-conduction parameter, $a=\left(N_{1}\right)_{0} v \mathrm{Re}^{1 / 2} / L$ is hydrodynamic slip parameter.

Examination of Eqns. (16)-(19) reveals some interesting special cases. In the absence of species diffusion i.e. neglecting the concentration equation and also negating heat source/sink effect $(G=0)$ and momentum slip $(a=0)$, taking the isothermal $(N c \rightarrow \infty)$, non-radiating $(N r=0)$ plate scenario, in the absence of free stream $(\lambda=0)$ and under the following minor modification:

$f=2^{3 / 2} F, \theta(\eta)=\theta(\xi), \eta=2^{1 / 2} \xi$

Eqns. (16)-(18) reduce to:

$F^{\prime \prime}+3 F F^{\prime \prime}-2 F^{\prime 2}+\theta=0$

$\theta^{\prime \prime}+3 \operatorname{Pr} F \theta^{\prime}=0$

The simplified boundary conditions (19) contract to:

$F(0)=F^{\prime}(0)=\theta(0)-1=F^{\prime}(\infty)=\theta(\infty)=0$.

Eqns. (21)-(22) with the boundary conditions Eqn. (23) correspond to the classical model of Ostrach ${ }^{[42]}$ and provide an excellent benchmark for validation of the numerical solutions.

\subsection{Quantities of physical interest}

The physical parameters of interest in the present problem are the skin friction factor (surface shear stress), $C_{f \bar{x}}$, the local Nusselt number (surface heat transfer rate) $N u_{\bar{x}}$ and the local Sherwood number (surface species diffusion rate) $S h_{\bar{x}}$ all of which are derived from spatial 
gradient functions of velocity, temperature and concentration, respectively. The appropriate expressions are:

$$
C_{f \bar{x}}=\frac{\mu}{\rho \bar{u}_{e}^{2}(\bar{x})}\left(\frac{\partial \bar{u}}{\partial \bar{y}}\right)_{\bar{y}=0}, \quad N u_{\bar{x}}=\frac{\bar{x}}{T_{f}-T_{\infty}}\left(-\frac{\partial T}{\partial \bar{y}}\right)_{\bar{y}=0}, \quad S h_{\bar{x}}=\frac{\bar{x}}{C_{w}-C_{\infty}}\left(-\frac{\partial C}{\partial \bar{y}}\right)_{\bar{y}=0} .
$$

Using Eqns. (6), (14), (15), we have from Eqn. (24):

$\operatorname{Re}_{\bar{x}}^{1 / 2} C_{f \bar{x}}=f^{\prime \prime}(0), \quad \operatorname{Re}_{\bar{x}}^{-1 / 2} N u_{\bar{x}}=-\theta^{\prime}(0), \quad \operatorname{Re}_{\bar{x}}^{-1 / 2} S h_{\bar{x}}=-\phi^{\prime}(0)$,

where $\operatorname{Re}_{x}=\bar{u}_{e} \bar{x} / v$ is the local Reynolds number.

\section{RESULTS AND DISCUSSION}

Equations (16)-(18) with the boundary conditions (19) were solved numerically Runge-KuttaFehlberg fourth-fifth order numerical method from Maple 17 software. The step size was assumed to be 0.001 and the convergence criteria was taken as $10^{-6}$. The asymptotic boundary conditions, given by Eqn. (19), were replaced by using a value of 12 for the similarity variable $\eta_{\max }$ as follows:

$\eta_{\max }=12, f^{\prime}(12)-1=\theta(12)=\phi(12)=0$.

The choice of $\eta_{\max }=12$ ensures that all numerical solutions approached the asymptotic values correctly. In order to validate the accuracy of the Maple numerical quadrature solutions, we compare our results with those of Bejan ${ }^{[35]}$. The comparison is shown in Table 1 and excellent agreement is clearly achieved. In order to further justify the correctness of the present computations, Eqns. (21)-(22) subject to boundary conditions (23) have also been solved and solutions compared with Ostrach ${ }^{[42]}$. Again excellent correlation is observed in Table 2. Confidence in the present results is therefore high. 
Table 1. Comparison of heat transfer rates for various Prandtl numbers with $N c \rightarrow \infty$.

\begin{tabular}{|c|c|c|}
\hline Pr & $\begin{array}{l}\text { Present } \\
-\theta^{\prime}(0) \operatorname{Pr}^{-1 / 4}\end{array}$ & Bejan \\
& & Nu $\mathrm{Ra}^{-1 / 4}$ \\
\hline 0.01 & 0.180 & 0.162 \\
\hline 0.72 & 0.387 & 0.387 \\
\hline 1 & 0.401 & 0.401 \\
\hline 2 & 0.426 & 0.426 \\
\hline 10 & 0.464 & 0.465 \\
\hline 100 & 0.489 & 0.490 \\
\hline 1000 & 0.497 & 0.499 \\
\hline
\end{tabular}

Table 2. Comparison of surface shear stress and heat transfer rate for different Prandtl numbers.

\begin{tabular}{|l|l|l|l|l|}
\hline & \multicolumn{2}{|c|}{$F^{\prime \prime}(0)$} & \multicolumn{2}{c|}{$-\theta^{\prime}(0)$} \\
\hline $\operatorname{Pr}$ & Ostrach $^{[42]}$ & Present & Ostrach & \\
\hline 0.72 & 0.6760 & 0.67602 & 0.5046 & 0.50463 \\
\hline 1 & 0.6421 & 0.64219 & 0.5671 & 0.56715 \\
\hline 10 & 0.4192 & 0.41919 & 1.1694 & 1.16933 \\
\hline
\end{tabular}

Graphical results for the variation of the dimensionless velocity, temperature and concentration (species diffusion) fields with distance transverse to the plate are provided in Figs. 2-16. Generally we prescribed the following parameter values: $N=0.5, a=G=N c=N r=0.1, \operatorname{Pr}=$ 0.72 (air), $S c=0.24$ (Hydrogen), unless otherwise stated. These correspond to buoyancyassisted flow with thermal buoyancy force being twice the magnitude of species (concentration) buoyancy force, weak slip, weak heat generation, weak convective boundary condition, weak thermal radiative flux compared with thermal conduction heat transfer, carrier fluid of air and hydrogen gas as the diffusing species ${ }^{[41]}$. Since both $S c$ and $\operatorname{Pr}$ are less than unity, both species diffusivity and thermal diffusivity will exceed momentum diffusivity. Furthermore throughout the computations we consider the case of mixed convection i.e., $\lambda=1$ and there is moving free stream (velocity at the boundary layer edge is non-zero), as reflected in the free stream boundary condition in Eqn. (19). 
Figs. 2-6 illustrate the influence of the governing thermophysical parameters on velocity evolution in the boundary layer. With an increase in convection-conduction parameter $(N c)$ there is a significant acceleration in the boundary layer flow (Fig. 2) in close proximity to the plate. A prominent peak arises near the plate and this is progressively displaced closer to the plate as $N c$ is increased. As anticipated further from the plate the influence of $N c$ vanishes. $N c$ embodies the relative influence of thermal convection heat transfer to thermal conduction heat transfer. For $N c$ $>1$. 0 , thermal convection dominates thermal conduction. For $N c<1$ thermal conduction is more significant than thermal convection. In consistency with this the maximum flow velocity is observed for the highest value of $N c$ i.e. 1.0. Fig. 3 reveals that a significant acceleration in the flow is achieved with an increase in momentum slip parameter. This was also noted by Sahoo and Do ${ }^{[43]}$ even for viscoelastic flows. As with the convection-conduction parameter, $N c$, momentum slip arises only in the boundary conditions (19) where the velocity boundary condition is equal to the product of momentum slip and shear stress at the plate. For the no-slip case, $a=0$ and clearly velocity is minimized. A velocity overshoot appears for all profiles near the plate and progressively migrates closer to the plate with increasing momentum slip. Similar observations have been reported by Mahmoud ${ }^{[11]}$. The strong influence of slip at the wall is of significance in the efficiency of materials processing operations. Inspection of Fig. 3 also indicates that the slip velocity at the wall is greater than zero for $a>0$. In accordance with slip flow theory, fluid velocity at the wall is also different from the wall velocity in proportion to the local velocity gradient normal to the wall, although this requires much finer resolution of the boundary layer structure and is beyond the scope of the present discussion. Fig. 4 depicts the velocity field response to a variation in radiation-conduction parameter; $\mathrm{Nr}$. $\mathrm{Nr}$ represents the relative contribution of thermal radiation to conduction heat transfer. For $N r=1$ both modes of heat transfer have the same contribution as described by Modest ${ }^{[25]}$. For $\mathrm{Nr}<1$ thermal conduction dominates over thermal radiation flux. For $\mathrm{Nr}>1$ thermal radiation contributes more than thermal conduction. All three cases are computed in Fig. 4. Evidently velocities are minimized for $\mathrm{Nr}=0$ for which thermal radiation flux vanishes. Increasing $\mathrm{Nr}$ accelerates the flow in particular in the vicinity of the near-wall regime. The increasing contribution of thermal radiation serves to augment the thermal diffusivity of the fluid regime which supplements thermal energy in the boundary layer and this boosts the velocity. Boundary layer thickness is 
therefore enhanced with increasing thermal radiation. Velocity overshoots are also observed in Fig. 4 as with Figs. 2 and 3. In all these figures the buoyancy-aided case $(N>0)$ is studied and backflow never arises i.e. velocity magnitudes are always positive everywhere in the boundary layer. In Fig. 5, we have computed the response of the velocity field with a change in heat generation parameter $(G)$ for the buoyancy-opposed case, $N=-1$. This implies that the species buoyancy force is in the opposite direction to thermal buoyancy force. In Fig. 5 momentum wall slip is also negated $(a=0)$. Even a slight increase in the heat source effect leads to a marked elevation in velocity magnitudes. In contrast to Figs. 2-4, we observe that a velocity shoot is absent. The velocity profiles all grow monotonically from the plate to a maximum in the free stream. Flow reversal also does not arise anywhere. The addition of thermal energy to the flow regime with increasing $G$ values effectively serves to accelerate the flow. Fig. 6 shows that a positive increase in buoyancy ratio parameter, $N$ strongly accentuates the flow near the plate whereas the converse response is induced with a negative increase in $N$. The parameter, $N$, strong couples the momentum field to the concentration field via the linear body force term, $N \phi$ arising in eqn. (16). When both thermal and species buoyancy forces are acting in unison $(N>0)$ the momentum is boosted considerably in the boundary layer and velocity shoots are computed. However when buoyancy forces are opposing each other, the flow is decelerated markedly and velocity overshoots vanish.

Figs. 7-11 depict the distributions of temperature in the boundary layer with various parameters. A marked increase in temperature is generated with a rise in convection-conduction parameter $(N c)$ as observed in Fig. 7. The maximum enhancement is evidently at the plate. Thermal boundary layer thickness will therefore be increased. Conversely a noticeable reduction in temperature is caused with an increase in momentum slip parameter (Fig. 8). Slip velocity has been shown to increase heat transfer rates from the bounding surface to the fluid as elucidated in Das ${ }^{[24]}$. This is due to an increase in advection in a region where diffusion is dominant. This has also been observed by Turkyilmazoglu ${ }^{[7]}$. Fig. 9 reveals that increasing presence of thermal radiation (i.e. larger values of $\mathrm{Nr}$ ) induces a substantial heating of the fluid regime and elevates thermal boundary layer thickness considerably. Thermal diffusivity of the fluid medium is augmented by thermal radiation, which aids in transport of heat into the boundary layer leading to temperature enhancement. A similar response was documented by Das ${ }^{[24]}$. Owing to the 
convective surface boundary condition at the plate $(N c=0.1)$ temperatures vary at the plate for each profile, but are clearly maximized for $N r=2$, for which thermal radiation contribution is double that of thermal conduction. An increase in heat source parameter $(G)$ in Fig.10 also aids thermal diffusion in the boundary layer and strongly increases temperatures both at the plate and for some distance transverse to it. Fig. 11 demonstrates that for buoyancy-opposed flow $(N<0)$ temperatures are increased whereas for buoyancy-assisted flow $(N>0)$ they are depressed. Thermal boundary layer thickness is therefore enhanced with increasingly vigorous buoyancy opposition.

Figs. 12-16 depict the distributions of the dimensionless concentration with various parameters. Concentration values are slightly depressed with increasing convection-conduction parameter $(N c)$ as shown in Fig. 12. Species diffusion is therefore opposed somewhat with increasing convection at the plate. Similarly a weak reduction in concentration is associated with a significant rise in momentum slip parameter (Fig. 13). Species diffusion is therefore most efficient in the regime for the no-slip case $(a=0)$. Concentration boundary layer thickness is therefore reduced with increasing velocity slip at the plate. Inspection of Figs 14, 15 shows that increasing thermal radiation $(N r)$ and heat generation $(G)$, there is a fall in species concentrations. With buoyancy-opposed flow $(N<0)$ the concentration boundary layer thickness is however enhanced (Fig. 16) whereas the opposite is apparent with buoyancy-aided flows $(N$ $>0$ ). In all the computations presented very smooth profiles have been achieved testifying to the satisfaction of convergence and the adequate specification of a far-field value of transverse coordinate, $\eta$.

\section{CONCLUSIONS}

Numerical solutions have been presented for the nonlinear buoyancy-driven mixed convection heat and mass transfer in slip flow from a vertical surface with thermal radiation and heat source effects. Lie group theory has been employed to derive similarity equations. Very good correlation between the present numerical method and earlier classical computations of Ostrach ${ }^{[42]}$ and Bejan ${ }^{[34]}$ have been obtained. It has been observed that:

(i) A rise in momentum slip parameter (a) elevates flow velocity but reduces temperature and concentration values. 
(ii) Increasing radiative-conductive ( $\mathrm{Nr}$ ) parameter increases velocity and temperature but lowers concentration.

(iii) With higher values of convection-conduction parameter $(N c)$ the velocity is enhanced as is fluid temperature whereas concentration of the diffusing species is decreased.

(iv) An increase in heat generation parameter $(G)$ leads to an acceleration of the flow and increasing temperature whereas it decreases concentration.

(v) Increasing positive values of buoyancy-ratio parameter $(N)$ corresponding to opposing flow accelerates the flow but reduces temperature and concentration; the converse response is computed for increasingly negative values of $N$ (buoyancyopposed flow).

The present study has considered Newtonian fluids. Future efforts will examine double-diffusive slip flows of viscoelastic fluids ${ }^{[44]}$ and will be communicated imminently.

Acknowledgement: The authors acknowledge financial support from Universiti Sains Malaysia, RU Grant 1001/PMATHS/811252.

\section{REFERENCES}

[1] Wang, C.Y. Flow due to a stretching boundary with partial slip - an exact solution of the Navier-Stokes equations, Chem. Eng. Sci., 2002, 57:3745-3747.

[2] Mukhopadhyay, S., Effects of slip on unsteady mixed convective flow and heat transfer past a porous stretching surface, Nuc. Eng. and Des., 2011,41:2660-2665.

[3] Ireland, P.M. and G. J. Jameson, "Foam slip on surfaces of intermediate or low wettability", Chem. Eng. Sci., 2009, 64:3859-3867.

[4] Khaled, A.R.A. and K. Vafai, Analysis of oscillatory flow disturbances and thermal characteristics inside fluidic cells due to fluid leakage and wall slip conditions, J. Biomecha., 2004,37:721-729.

[5] Ulmanella U. and Ho, C.M. Molecular effects on boundary condition in micro/nanoliquid flows. Phys. Fluids 2008, 20, doi: 10.1063/1.3006031.

[6] Bocquet, L. and Barrat, J.L. Flow boundary conditions from nano- to micro-scales. Soft Matt. 2007, 3:685-693.

[7] Nandeppanavar, M.M., K. Vajravelu, M. Subhas Abel and M.N. Siddalingappa, Second order slip flow and heat transfer over a stretching sheet with non-linear Navier boundary condition, Int. J. Therm. Sci., 2012, 58:143-150. 
[8] Turkyilmazoglu, M. Slip flow and heat transfer over a specific wedge: an exactly solvable Falkner-Skan equation, J Eng Math (2015) 92:73-81.

[9] Tripathi, D., Anwar Bég, O. and Curiel Sosa, J.L. Homotopy semi-numerical simulation of peristaltic flow of generalized Oldroyd-B fluids with slip effects, Compu. Meth. in Biomec. and Biomed. Eng. (2012). DOI: 10.1080/10255842.2012.688109.

[10] Wang, C.Y, The effect of slip on the flow in a rotating channel, Chem. Eng. Commun., 2013, 200: 587-594.

[11] Mahmoud, M.A.A. Heat and mass transfer in stagnation-point flow towards a vertical stretching sheet embedded in a porous medium with variable fluid properties and surface slip velocity, Chem. Eng. Commun., 2013, 200:543-562.

[12] Taamneh, Y. and Omari, R. Slip-flow and heat transfer in a porous micro-channel saturated with power-law fluid, J. Fluids 2013(2013) Article ID 604893, 9 pages.

[13] Anand, V. Slip law effects on heat transfer and entropy generation of pressure driven flow of a power law fluid in a microchannel under uniform heat flux boundary condition, Energy, 2014, 76:716-732.

[14] Jamalabadi, M.Y.A., Park, J.H. and Lee, C.Y. Optimal design of magnetohydrodynamic mixed onvection flow in a vertical channel with slip boundary conditions and thermal radiation effects by using an entropy generation minimization method, Entropy 2015, 17:866-881 .

[15] Abbas, Z., Sheikh, M. and Pop, I. Stagnation-point flow of a hydromagnetic viscous fluid over stretching/shrinking sheet with generalized slip condition in the presence of homogeneousheterogeneous reactions, J. of the Taiwan Ins. of Chem. Eng. 2015: 1-7.

[16] Yu, Z. Liu, X. and Kuang, G. Water slip flow in superhydrophobic microtubes within laminar flow region, Chinese J. of Chem. Eng. In Press.

[17] Nandy, S.K. Unsteady flow of Maxwell fluid in the presence of nanoparticles toward a permeable shrinking surface with Navier slip, J. of the Taiwan Ins. of Chem. Eng. In Press.

[18] Haq, R.U., Nadeem, S., Khan, Z.H. and Akbar, N.S. Thermal radiation and slip effects on MHD stagnation point flow of nanofluid over a stretching sheet, Physica E 2015, 65:17-23.

[19] Ibáñez, G. Entropy generation in MHD porous channel with hydrodynamic slip and convective boundary conditions, Int. J. of Heat and Mass Transfer 2015, 80:274-280.

[20] Bég, O.A., Uddin, M.J., Rashidi, M.M., and Kavyani, N. Double-diffusive radiative magnetic mixed convective slip flow with Biot and Richardson number effects. Journal of Engineering Thermophysics, 2014,23:79-97. 
[21] Unal, C., Bohl, W.R. and Pasamehmetoglu, K.O. Modeling of heat and mass transfer in accelerator targets during postulated accidents. Nuc. Eng. Design, 196, 185-200 (2000).

[22] Rana, P., Bhargava, R. and Anwar Bég, O. Finite element simulation of unsteady magnetohydrodynamic transport phenomena on a stretching sheet in a rotating nanofluid, Proc. IMechEPart N: J. Nanoeng. and Nanosys. (2012). DOI:10.1177/1740349912463312.

[23] Zueco, J., Anwar Bég, O., Takhar, H.S. and Prasad, V.R. Thermophoretic hydromagnetic dissipative heat and mass transfer with lateral mass flux, heat source, Ohmic heating and thermal conductivity effects: Network simulation numerical study, App.Therm. Eng., 2009,29:28082815.

[24] Das, K. Slip effects on heat and mass transfer in MHD micropolar fluid flow over an inclined plate with thermal radiation and chemical reaction. Int. J. Num. Meth. Fluids 2012, 70: 96-113.

[25] Modest, M.F, Thermal Radiation Heat Transfer, MacGraw-Hill, New York, USA (1993).

[26] Mahmoud, M..A., Variable fluid properties and thermal radiation effects on mixed convection flow over a horizontal surface, Int. J. Com. Meth. Eng. Sci. Mech., 2010,11:299-303.

[27] Gupta, D., Kumar, L., Anwar Bég, O. and Singh, B. Finite element simulation of mixed convection flow of micropolar fluid over a shrinking sheet with thermal radiation, Proc IMechEPart E: J. Process Mecha. Eng. 2014, 228:1 61-72.

[28] Khan, W.A., M.J. Uddin and Ismail, A.I.M. Effects of radiation on Blasius slip flow of oxide nanofluids with Merkin boundary condition. Proc. IMechE- Part N: J. Nanoengineering and Nanosystems, 2012.

[29] Hussain, M., Ashraf, M., Nadeem, S. and Khan, M. Radiation effects on the thermal boundary layer flow of a micropolar fluid towards a permeable stretching sheet, J. of the Franklin Ins., 2013, 353:194-210.

[30] Mohankrishna, P., Sugunamma V. and Sandeep N. Radiation and magneticfield effects on unsteady natural convection flow of a nanofluid past aninfinite vertical plate with heat source. Chem Process Eng Res 2014;25:39-52.

[31] Srinivasacharya, D., Reddy, G.S. Chemical reaction and radiation effects on mixed convection heat and mass transfer over a vertical plate in power-law fluid saturated porous medium, J. of the Egyp. Math. Soc. In Press.

[32] Rajamohan, G., Narayanaswamy, R. and Perumal, K. Mixed Convection and Radiation Heat Transfer in a Horizontal Duct with Variable Wall Temperature. Heat Transfer Eng. 2015, 36:335-345. 
[33] Ashraf, M.B., Hayat, T., Alsaedi, A., Shehzad, S. A. Convective heat and mass transfer in MHD mixed convection flow of Jeffrey nanofluid over a radially stretching surface with thermal radiation, J. of Cen. South Uni., 2015,22: 1114-1123

[34] Pal, D., Mandal, G. Mixed convection-radiation on stagnation-point flow of nanofluids over a stretching/shrinking sheet in a porous medium with heat generation and viscous dissipation, J. of Pet. Sci. and Eng., 2015,126:16-25.

[35] Bejan, A, Chapter 4, Convection Heat Transfer, $4^{\text {th }}$ ed., Wiley, New York, (2013).

[36] Karniadakis, G., Beskok, A. and Aluru, N. Microflows and Nanoflows Fundamentals and Simulation. In: Microflows and Nanoflows Fundamentals and Simulation. Springer

Science, New York (2005)

[37] Uddin, M.J., Bég, O.A., Aziz, A., and Ismail, A.IMD. Group analysis of free convection flow of a magnetic nanofluid with chemical reaction. Math. Prob. in Eng. 2015, 2015, Article ID 621503, 11 pages.

[38] Hang, X. Lie group analysis of a nanofluid bioconvection flow past a vertical flat surface with an outer power-law stream. J. of Heat Transfer 2015, 137:041101.

[39] Rashidi, M.M., Rahimzadeh, N., Ferdows, M., Uddin M.J. and Anwar Bég, O. Group theory and differential transform analysis of mixed convective heat and mass transfer from a horizontal surface with chemical reaction effects, Chem. Eng. Commun., 2012, 199:1012-1043.

[40] Asghar, S., Jalil, M., Hussan, M. and Turkyilmazoglu, M. Lie group analysis of flow and heat transfer over a stretching rotating Disk. Int. J. of Heat and Mass Transfer 2014, 69:140-146.

[41] Cao, L., Si, X., Zheng, L. and Pang, H. Lie group analysis for MHD effects on the convectively heated stretching porous surface with the heat source/sink, Boundary Value, (2015) Problems, 2015,63.

[40] Ostrach, S., An analysis of laminar free-convection flow and heat transfer about a flat plate parallel to the direction of the generating body force, Nat. Adv. Comm. Aero., Report No. 1953, 1111:63-79.

[42] Bég, O. A., Prasad, V. R., Vasu, B., Reddy, N.B., Li , Q. and Bhargava, R. Free convection heat and mass transfer from an isothermal sphere to a micropolar regime with Soret/Dufour effects, Int. J. Heat and Mass Transfer, 2011,54:9-18.

[43] Sahoo, B. and Do, Y. Effects of slip on sheet-driven flow and heat transfer of a third grade fluid past a stretching sheet, Int. Comm. Heat Mass Transfer, 2010, 37:1064-1071. 
[44] Tripathi, D., and Anwar Bég, O. A numerical study of oscillating peristaltic flow of generalized Maxwell viscoelastic fluids through a porous medium, Transp. in Porous Media, 2012, 95:337-348.

\section{Nomenclature}

$a \quad$ hydrodynamic slip parameter (-)

A parameter of the group (-)

$c_{p} \quad$ specific heat at constant pressure $\left(\mathrm{J} \mathrm{kg}^{-1} \mathrm{~K}^{-1}\right)$

$C_{f \bar{x}} \quad$ local skin-friction coefficient (-)

C concentration $\left(\mathrm{kg} \mathrm{m}^{-3}\right)$

$C_{w} \quad$ wall concentration $\left(\mathrm{kg} \mathrm{m}^{-3}\right)$

$C_{\infty} \quad$ ambient concentration $\left(\mathrm{kg} \mathrm{m}^{-3}\right)$

$D \quad$ mass diffusion coefficient $\left(\mathrm{m}^{2} \mathrm{~s}^{-1}\right)$

$f(\eta)$ dimensionless stream function (-)

$G \quad$ heat generation/ absorption parameter (-)

$g \quad$ acceleration due to gravity $\left(\mathrm{ms}^{-2}\right)$

$h_{f} \quad$ heat transfer coefficient $\left(\mathrm{Wm}^{-2} \mathrm{~K}^{-1}\right)$

$k \quad$ fluid thermal conductivity $\left(\mathrm{Wm}^{-1} \mathrm{~K}^{-1}\right)$

$k_{1} \quad$ Rosseland mean absorption coefficient $\left(\mathrm{m}^{-1}\right)$

L characteristic length (m)

$N \quad$ buoyancy ratio parameter (-)

Nc convection-conduction parameter (-) 


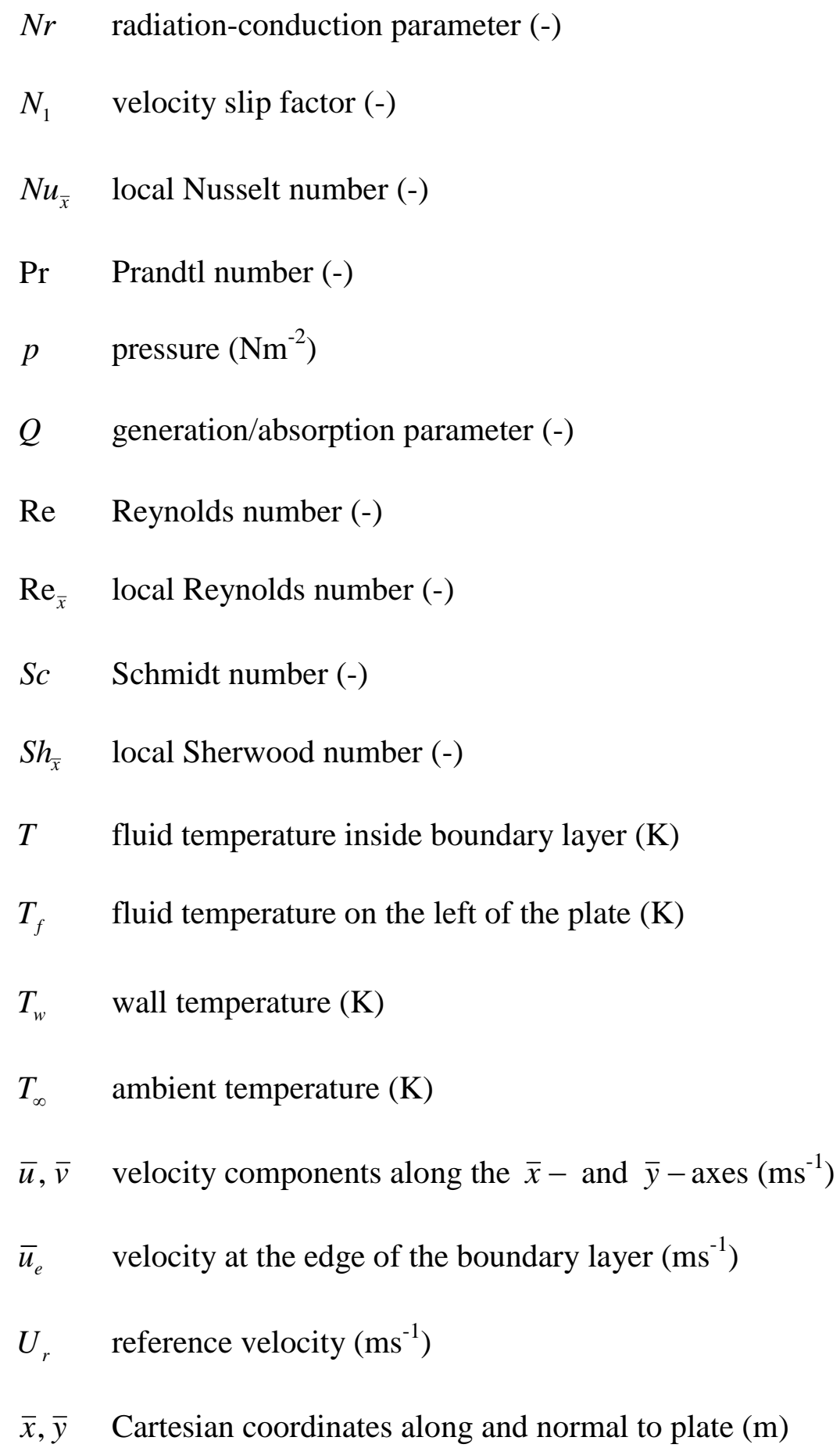

\section{Greek symbols}

$\alpha \quad$ thermal diffusivity $\left(\mathrm{m}^{2} \mathrm{~s}^{-1}\right)$

$\alpha_{i} \quad$ constants (-) 
$\beta_{T} \quad$ volumetric thermal expansion coefficient $\left(\mathrm{K}^{-1}\right)$

$\beta_{C} \quad$ volumetric solutal expansion coefficient $\left(\mathrm{K}^{-1}\right)$

$\mu \quad$ dynamic viscosity of the fluid $\left(\mathrm{Nsm}^{-2}\right)$

$\sigma_{1} \quad$ Stefan-Boltzmann constant $\left(\mathrm{Wm}^{-2} \mathrm{~K}^{-4}\right)$

$v \quad$ kinematic viscosity of the fluid $\left(\mathrm{m}^{2} \mathrm{~s}^{-1}\right)$

$\phi(\eta)$ dimensionless concentration function

$\eta \quad$ independent similarity variable (-)

$\theta(\eta)$ dimensionless temperature (-)

$\rho \quad$ fluid density $\left(\mathrm{kgm}^{-3}\right)$

$\psi \quad$ stream function (-) 


\section{Figures Captions}

Fig. 1 Flow configuration and coordinate system

(a) heat transfer from wall to fluid, (b) heat transfer from fluid to wall.

Fig.2: Effect of convection-conduction parameter on the dimensionless velocity.

Fig.3: Effect of momentum slip parameter on the dimensionless velocity.

Fig.4: Effect of radiation-conduction parameter on the dimensionless velocity.

Fig.5: Effect of heat generation (source) parameter on the dimensionless velocity.

Fig.6: Effect of buoyancy ratio parameter on the dimensionless velocity.

Fig.7: Effect of convection-conduction parameter on the dimensionless temperature.

Fig.8: Effect of momentum slip parameter on the dimensionless temperature.

Fig.9: Effect of radiation-conduction parameter on the dimensionless temperature.

Fig. 10: Effect of heat generation (source) parameter on the dimensionless temperature.

Fig. 11: Effect of buoyancy ratio parameter on the dimensionless temperature.

Fig. 12: Effect of convection-conduction parameter on the dimensionless concentration.

Fig. 13: Effect of momentum slip parameter on the dimensionless concentration.

Fig. 14: Effect of radiation-conduction parameter on the dimensionless concentration.

Fig.15: Effect of heat generation (source) parameter on the dimensionless concentration.

Fig.16: Effect of buoyancy ratio parameter on the dimensionless concentration. 


\section{$\underline{\text { Figures }}$}
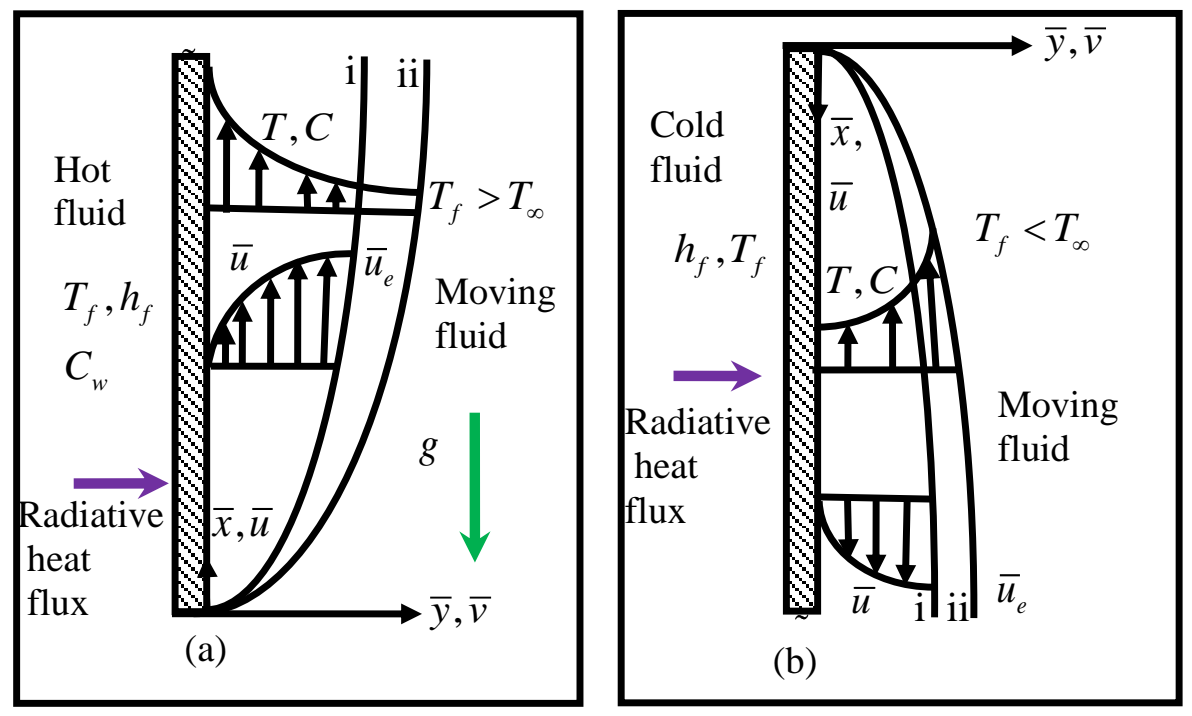

Fig.1

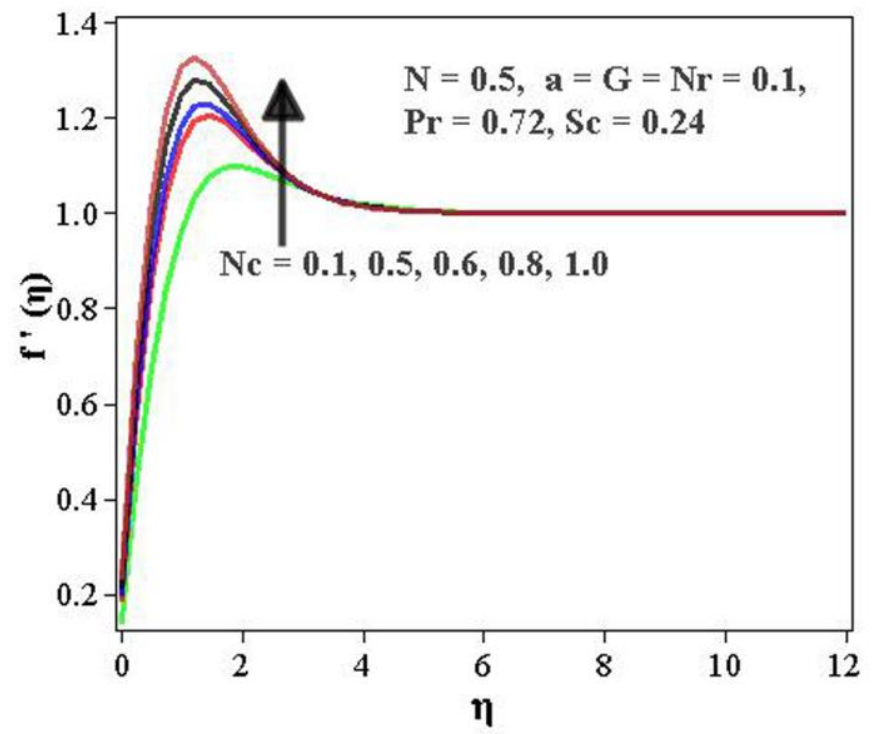

Fig.2 


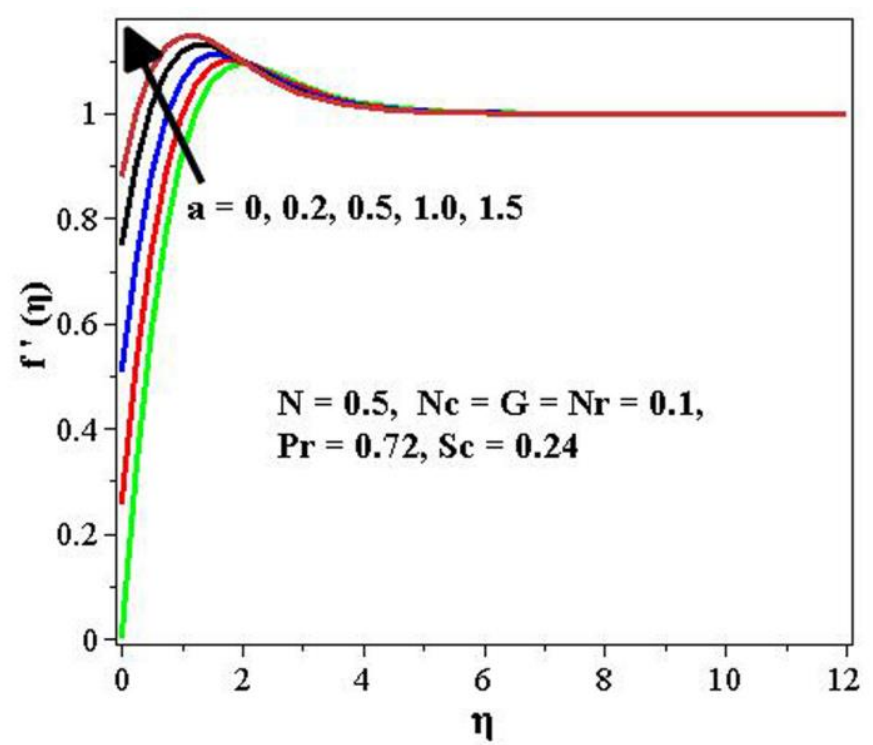

Fig.3

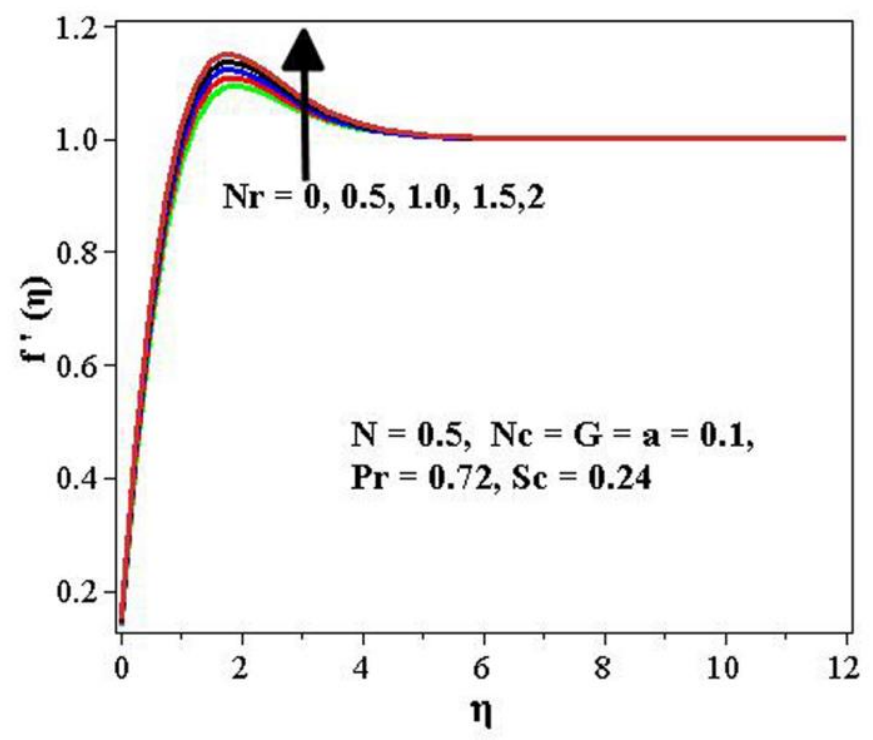

Fig.4 


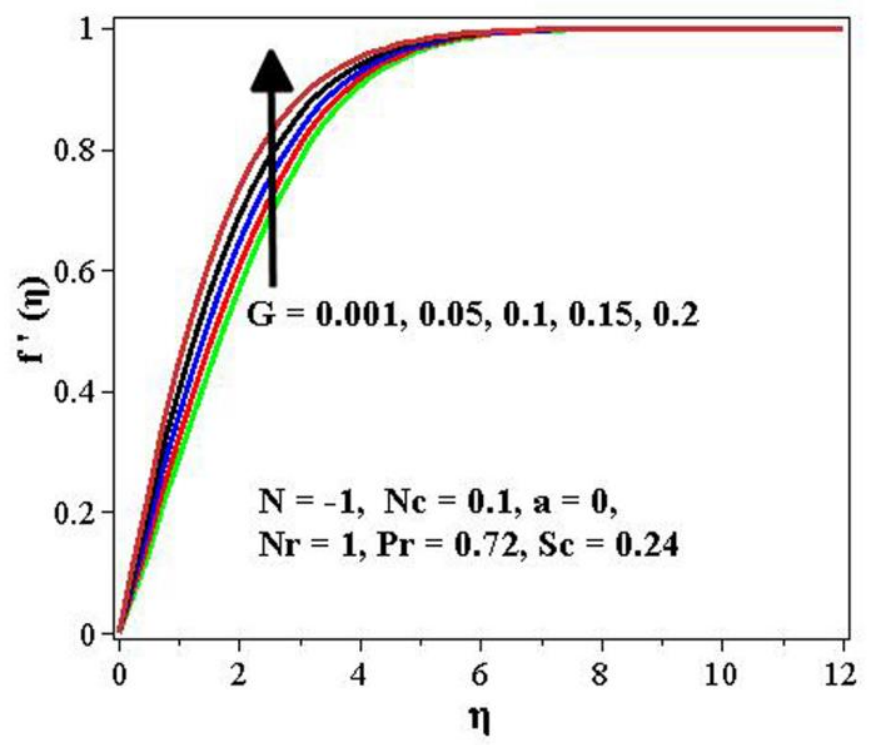

Fig.5

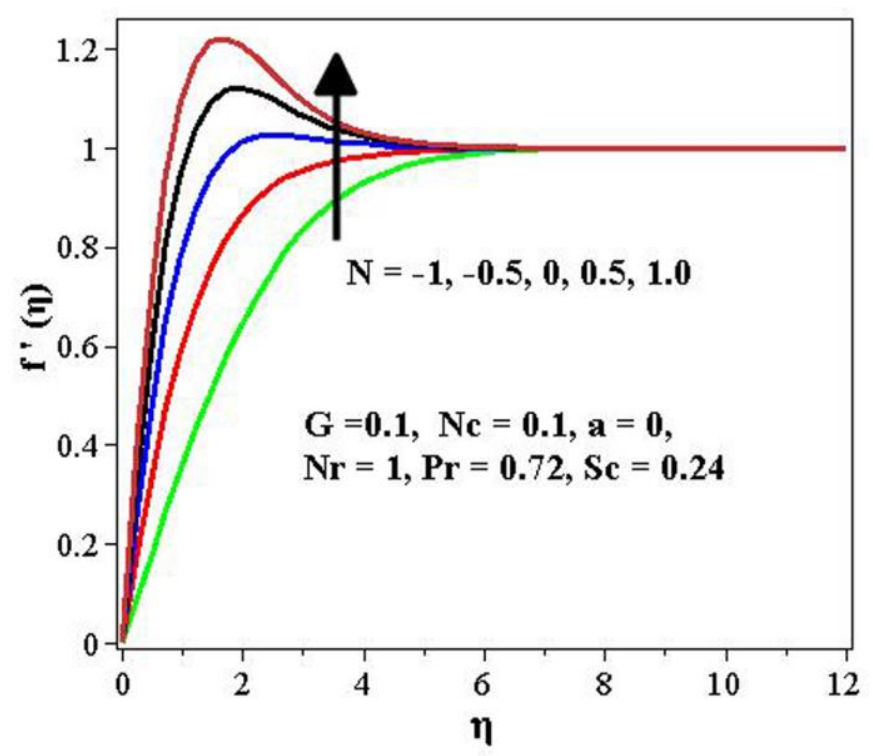

Fig.6 


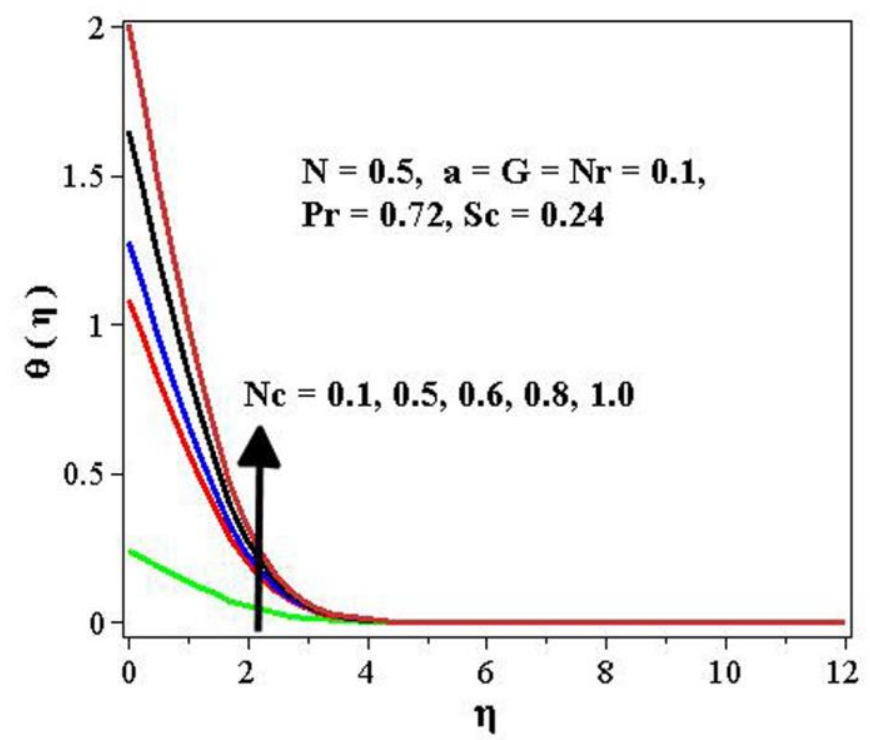

Fig.7

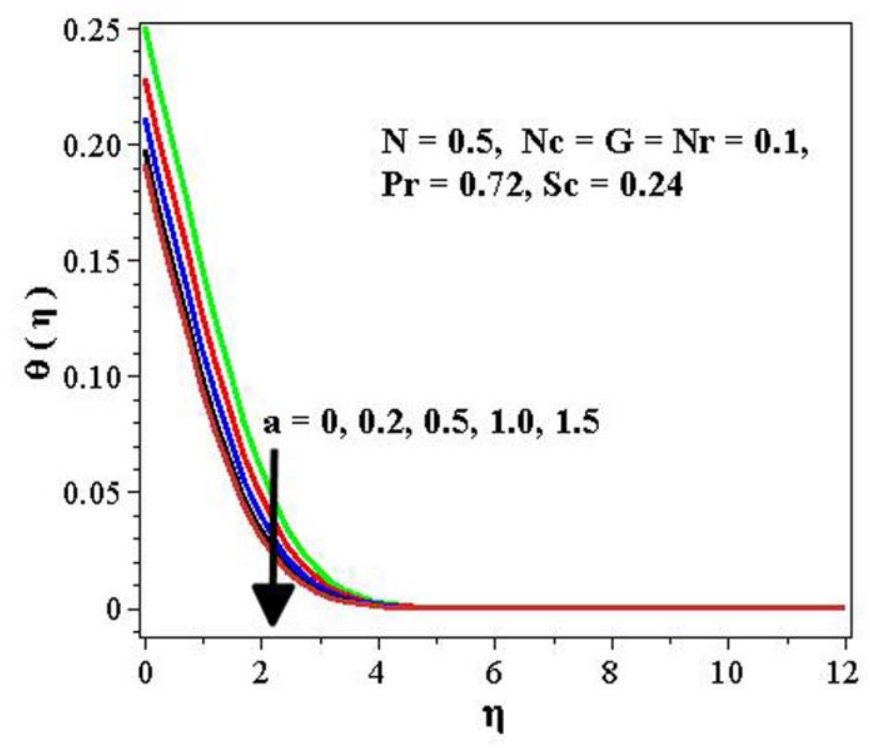

Fig.8 


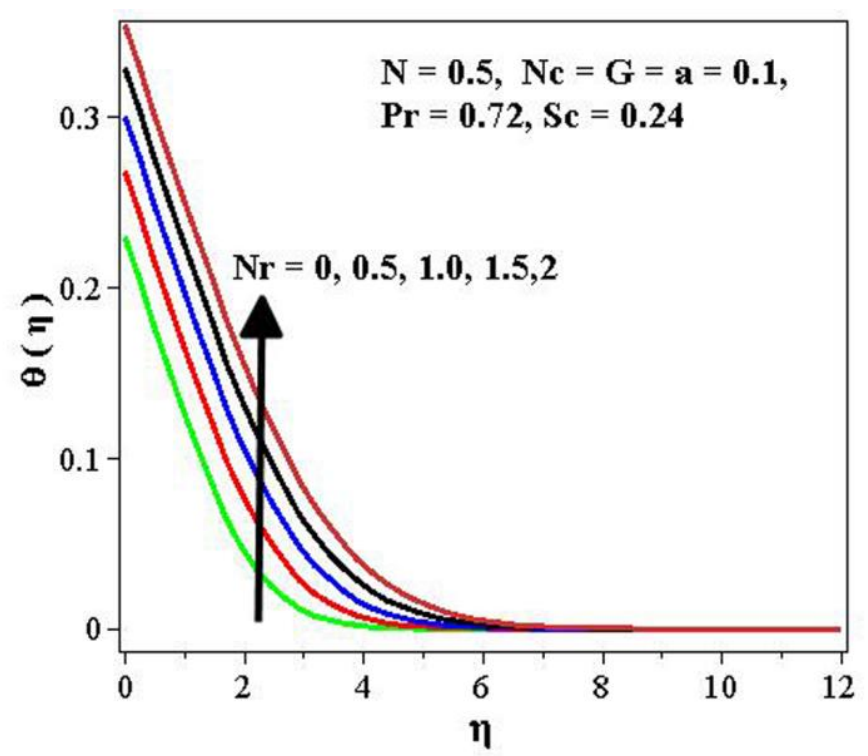

Fig.9

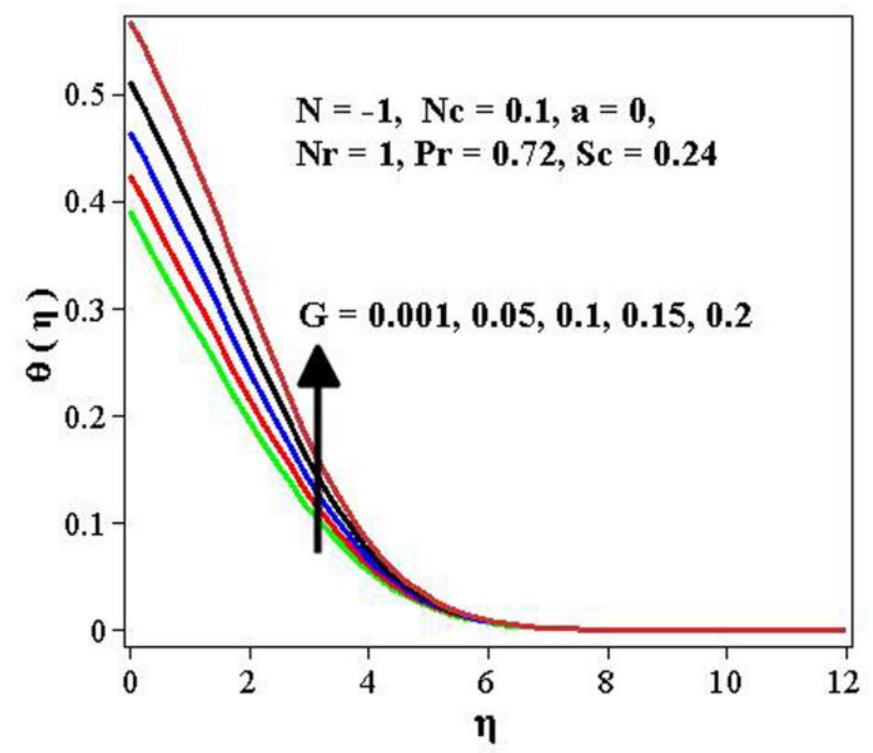

Fig.10 


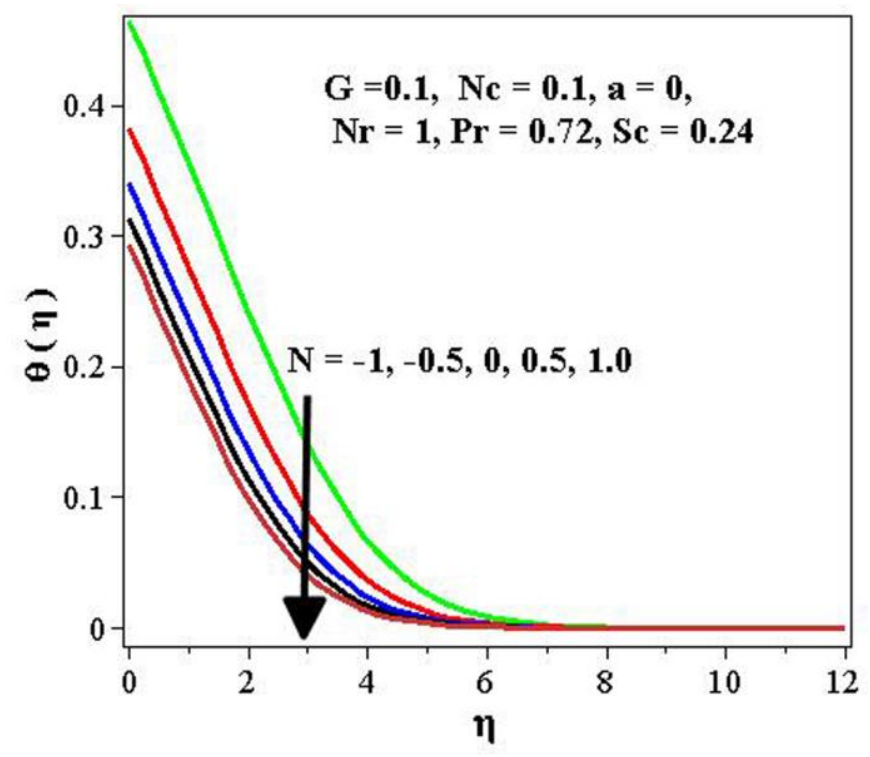

Fig.11

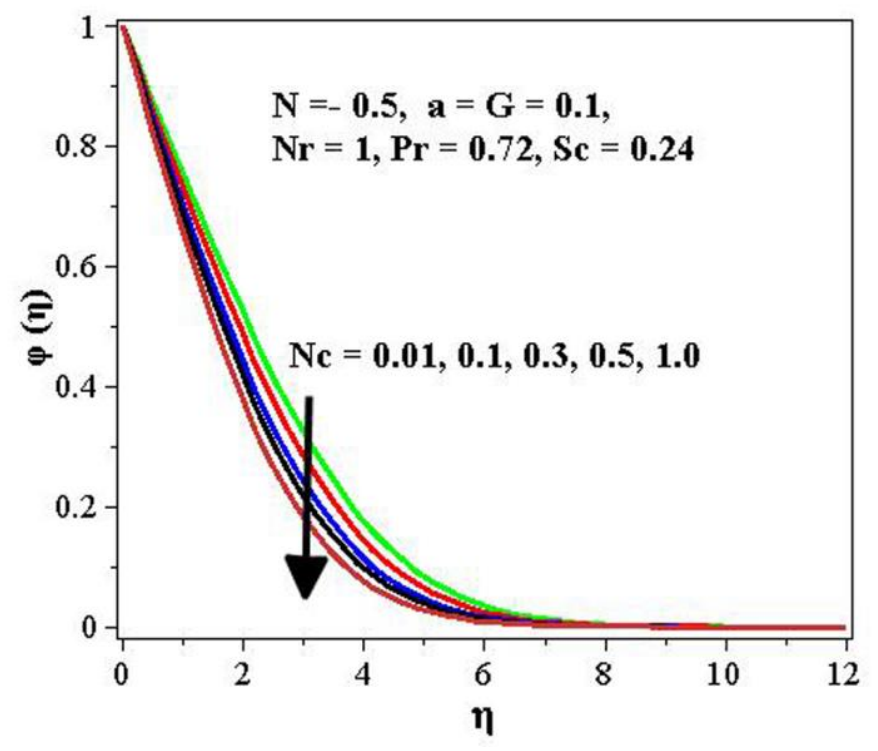

Fig.12 


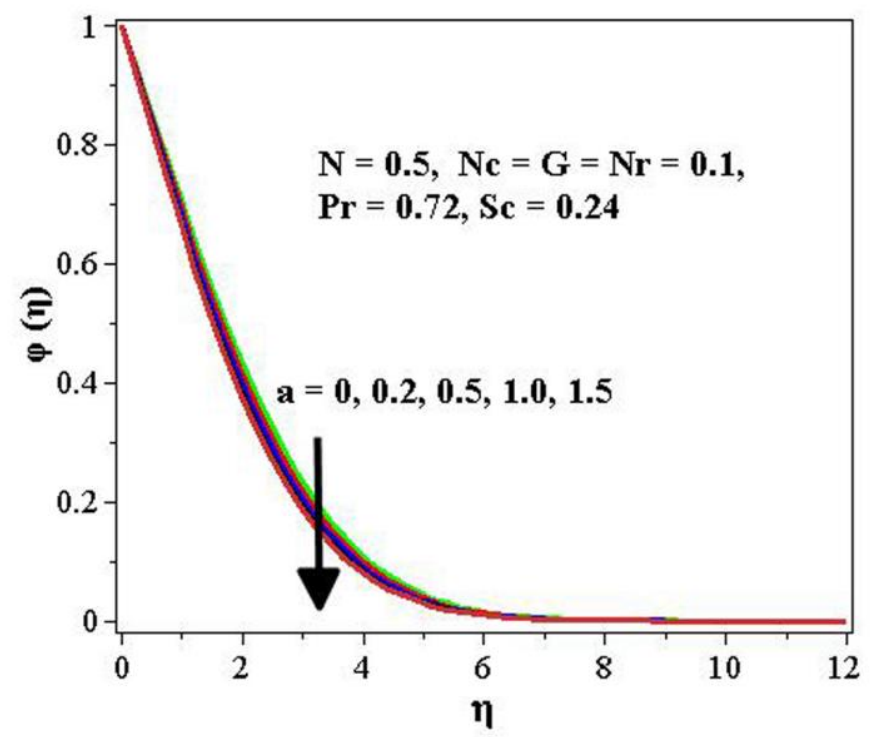

Fig.13

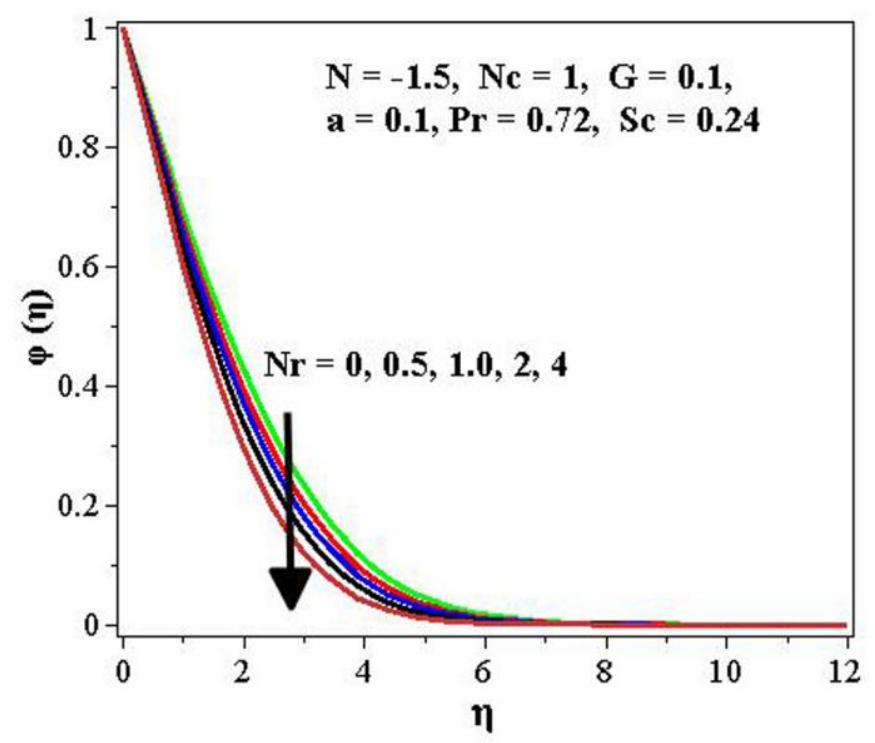

Fig.14 


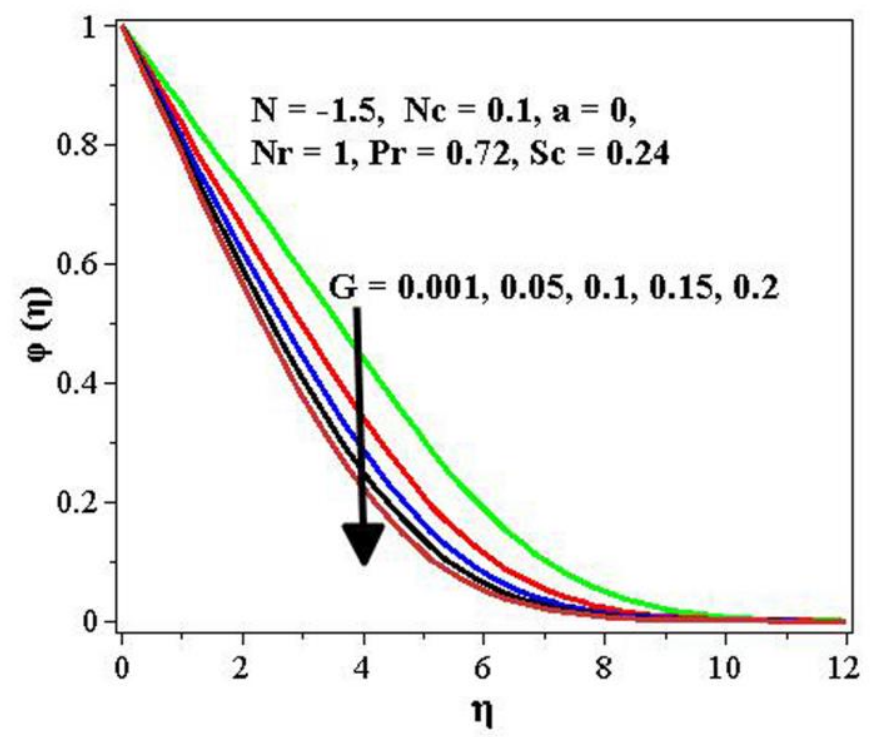

Fig.15

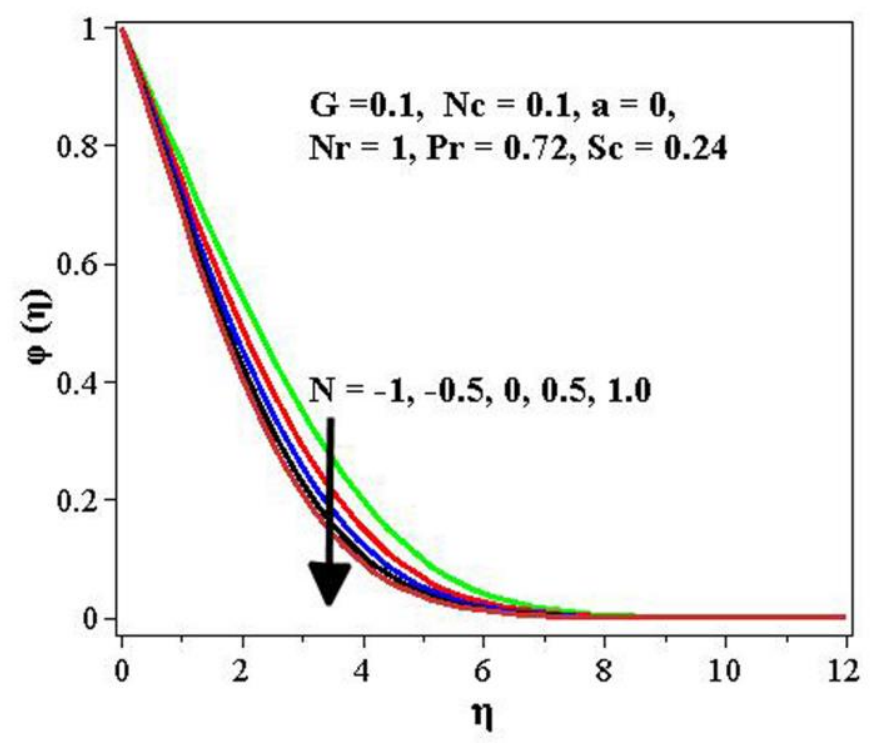

Fig.16 